\title{
Online Domain Adaptation for Continuous Cross-Subject Liver Viability Evaluation Based on Irregular Thermal Data
}

\author{
Sahand Hajifar ${ }^{\mathrm{a}}$ and Hongyue Sun ${ }^{\mathrm{b}}$ \\ ${ }^{\mathrm{a}, \mathrm{b}}$ Department of Industrial and Systems Engineering, University at Buffalo, Buffalo, NY \\ 14260, USA
}

ARTICLE HISTORY

Compiled November 26, 2020

\begin{abstract}
Accurate evaluation of liver viability during its procurement is a challenging issue and has traditionally been addressed by taking invasive biopsy on liver. Recently, people have started to investigate on the non-invasive evaluation of liver viability during its procurement using the liver surface thermal images. However, existing works include the background noise in the thermal images and do not consider the cross-subject heterogeneity of livers, thus the viability evaluation accuracy can be affected. In this paper, we propose to use the irregular thermal data of the pure liver region, and the cross-subject liver evaluation information (i.e., the available viability label information in cross-subject livers), for the real-time evaluation of a new liver's viability. To achieve this objective, we extract features of irregular thermal data based on tools from graph signal processing (GSP), and propose an online domain adaptation (DA) and classification framework using the GSP features of cross-subject livers. A multiconvex block coordinate descent based algorithm is designed to jointly learn the domain-invariant features during online DA and learn the classifier. Our proposed framework is applied to the liver procurement data, and classifies the liver viability accurately.
\end{abstract}

\section{KEYWORDS}

Irregular Data; Graph Signal Processing; Liver Viability; Online Domain Adaptation; Real-time Classification

CONTACT Hongyue Sun. Email: hongyues@buffalo.edu 


\section{Introduction}

According to the Organ Procurement and Transplantation Network, there are over 120,000 patients needing an organ transplant as of Sep. 3rd, 2020 (HRSA, 2020). Every 9 minutes, someone is added to the national transplant waiting list, and 95 transplants take place each day in the US on average (HRSA, 2020). One challenge for the organ transplantation is that the maximum viability of organs under ideal procurement conditions is limited, only 4-6 hours for lungs and hearts and 8-12 hours for livers and pancreas. The actual viability of an organ is dependent on many factors such as donor health and procurement conditions. It is very important to accurately evaluate an organ's viability before its transplantation.

In this paper, we focus on the liver disease. According to the National Center for Health Statistics, there are 4.5 million adults being diagnosed with liver disease in 2018 (CDC, 2020). An early diagnosis and treatment of liver disease can be challenging (Mueller et al., 2014; Badebarin et al., 2017), and many patients eventually suffer from end-stage liver disease. For end-stage liver disease, which accounts for 41,743 deaths in the US in 2017, liver transplantation has proven to be the most effective method to extend patients' life (CDC, 2020; Lan et al., 2018). However, after a liver being harvested from a donor, it needs to wait for a matching recipient and be transported to the recipient. During this period, the liver is usually kept in a cold box with circulating perfusion fluid for hours to reduce the decrease of functionality (Cameron and Cornejo, 2015, Lan et al., 2018). Before transplantation, pathologists need to evaluate the liver viability and make sure it is safe to transplant the liver.

Currently, there are mainly two approaches being used to evaluate the liver viability: visual inspection and biopsy (Keeffe, 2001; Lan et al., 2015). In visual inspection, the pathologists look at the surface of a liver and judge its viability. The visual inspection may suffer from the pathologists' inadequate experiences and is subject to the lack of consistent standards (Petrick et al. 2015$)$. In addition, the appearances of a viable and an unviable organ may not be distinguishable (Gao et al., 2020). On the other hand, the biopsy examination and histologic analysis technique was initially used in organ transplantation in the 1980s, and has proven to be a more accurate evaluation technique (Vazquez-Martul and Papadimitriou, 2004). However, the biopsy examination technique suffers from two disadvantages: (1) the judgment on biopsy results depends on the pathologists' expertise and is prone to the subjective errors; and (2) taking biopsy is an invasive procedure and causes very small but irremediable damage to the organ (Rothuizen and Twedt, 2009, Lan et al., 2015). It was reported that the lack of accurate non-invasive assessment methods wastes $20 \%$ of donor organs every year (Lan, 2020).

In recent years, infrared thermography (IRT) has gained considerable attention in medical applications as a fast, passive, non-contact and non-invasive measuring technique (Lahiri et al., 2012). For instance, a research team from Virginia Tech designed a machine perfusion and corresponding non-invasive thermal imaging system to measure the surface temperature of livers, as shown in Figure 1 (a). Several analyses were performed for the liver viability assessment, and revealed a close relationship between the organ surface temperatures and viability based on this system (Lan et al., 2015, 2018, Gao et al., 2018, 2020). As will be discussed in detail in the literature review, existing works on thermal imaging based organ viability evaluation have the limitations of: (1) using the full thermal images and thus including the background data in addition to the pure liver region (shown in Figure 1(b)) in the analysis. The background data may

vary over time, and are irrelevant to the liver viability evaluation; (2) only considering 
the data in one liver or directly combining data from multiple livers in the analysis without considering the cross-subject liver heterogeneity. The variations in donors and perfusion conditions bring in the heterogeneous thermal images among livers, which need to be explicitly considered in the analysis; (3) cannot be used for the online crosssubject liver viability evaluation. The thermal images are collected continuously over time, and a model needs to be updated to accommodate for the latest information for the liver being evaluated, which will allow for the accurate online viability evaluation.



(a)
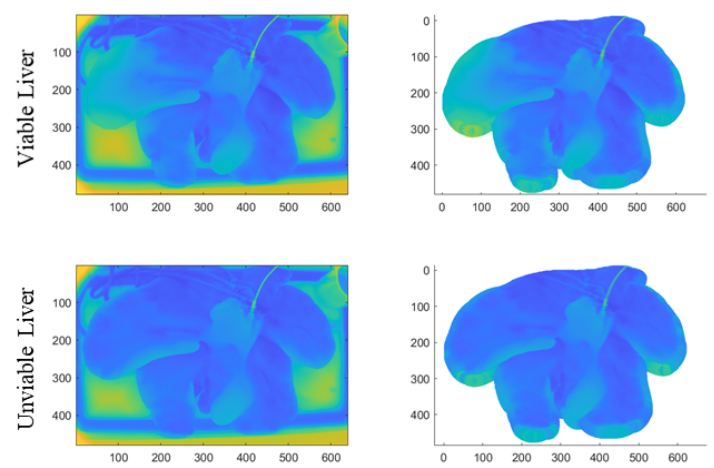

(b)

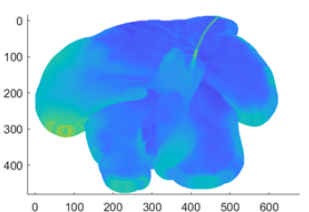

Figure 1. (a) Perfusion and non-invasive thermal imaging system (Lan 2020). Note that biopsy extraction was not a part of this work and was used for the purpose of viability evaluation; (b) Thermal images (on the left) of a viable liver versus an unviable liver, and the corresponding irregular pure liver regions (on the right)

The objective of this paper is to design an online non-invasive liver viability evaluation framework based on the pure liver region thermal data and cross-subject evaluation information. This framework leverages the thermal data and biopsy evaluation information from heterogeneous livers, to predict the viability of a new liver based on its thermal images in real time without the need of taking biopsies. To achieve this objective, we face several challenges: (1) The pure liver region is irregular (irregular data format), where traditional feature and image processing methods are not directly applicable; (2) The heterogeneity of livers, because of different donors and machine perfusion conditions, prevent us from directly training a model for cross-subject liver viability evaluation; and (3) The continuously collected thermal images contain rich information on the liver condition, and one needs to update the model with the streaming images while still achieve the online viability evaluation. To address these challenges, we propose a novel online domain adaptation (DA) and classification framework based on the graph signal processing (GSP) features of irregular pure liver thermal data. The contributions of the proposed framework are:

- We propose to model the irregular thermal data of the pure liver region by graph signal processing for the first time, and use graph Fourier transform (GFT) to extract the graph features from the irregular data.

- We propose a novel online DA and classification framework based on the graph features for the online cross-subject liver viability evaluation.

- We develop a novel multiconvex block coordinate descent based algorithm to jointly learn the domain-invariant features and the classifier.

The reminder of the paper is organized as follows. In Section 2, we review the relevant literature on organ viability evaluation based on thermal images, on GSP and on DA. Our proposed method is introduced in Section 3, and being demonstrated in 
Section 4. Finally, we conclude the paper and discuss about the future work in Section 5 .

\section{Literature Review}

\subsection{Organ Viability Evaluation based on Thermal Images}

IRT has been applied to a variety of applications including, but not limited to, kidney transplantation, brain imaging, breast cancer and liver disease (Lahiri et al., 2012). Researchers have revealed a close association between the histomorphologic quality and surface temperatures of the organ (Gorbach et al. 2009; Skowno and Karpelowsky, 2014; Vidal et al., 2014; Lan et al., 2015; Kochan et al., 2015; Lan et al., 2018; Gao et al., 2018, 2020).

Based on the collected data, different statistical methods can be utilized for organ viability evaluation. Cox regression models were applied to determine the main factors that would result into liver failure in the post-transplant period (Feng et al., 2006). A hierarchical regression method was utilized to estimate donor risk index based on patient and transplant center characteristics (Volk et al., 2011). Lan et al. (2015) applied quantitative and qualitative models to predict the number of dead cells in a liver and viability of the liver. Lan et al. (2018) extracted the principal components from the thermal images, and performed intra liver classification with logistic regression and inter liver classification with multitask learning. The classification can distinguish the viable versus unviable images within a liver accurately but did not generalize well to multiple livers since it did not consider the cross-subject heterogeneity among livers. The above works did not consider the updated liver information over time thus cannot achieve online viability evaluation. Gao et al. (2018, 2020) performed the change detection of surface temperature variation for a single liver in an online manner, and found that the temperature measurements show a high variation when the liver is viable and a low variation if the liver is unviable. However, these works only detected the change for a single liver and did not borrow the valuable pathologists' evaluation information from other livers, which can be beneficial to the viability evaluation.

There is a lack of online organ viability evaluation method to explicitly consider the heterogeneous livers' thermal images and evaluation information, and to address the irregular thermal data of the pure liver region.

\subsection{Graph Signal Processing}

GSP is a quickly developing subfield of signal processing that generalizes classical techniques of signal processing to irregular graph domain by considering the graph structure of multivariate signals (Ménoret et al. 2017). According to Ortega et al. (2018), GSP has seen major applications in sensor networks, image and 3-D point cloud processing, and biological networks.

We review the related research on biological networks, where many works modeled the data as a network (i.e., graph), such as the human brain (Ortega et al., 2018; Tran et al., 2019). Unlike in classical signal processing (Sartipi et al., 2020 Kang et al., 2020), GSP aims at learning and leveraging the underlying graph structure of the brain (Ortega et al. , 2018). In particular, the human brain activity signals can be modeled as a graph structure, where nodes and edge weights represent brain regions and structural connectivity between brain regions, respectively (Sporns, 2010, Bullmore and Sporns, 
2012). Hu et al. (2016a) modeled the progression of brain atrophy through a diffusion model over the brain connectivity graph. Hu et al. (2016b) proposed a matched signal detection theory to be applied to the neuroimaging data classification problem of Alzheimer's disease. Pirayre et al. $(2015,2017)$ used GSP to infer gene interactions, which play a vital role in the recognition of novel regulatory processes in cells. The application of GSP in other biological networks is not explored yet and still remains a research gap.

In this paper, we will use the tools in GSP for the feature extraction of the irregular thermal data of pure liver region.

\subsection{Domain Adaptation}

DA, a subfield of transfer learning, can transfer the information learnt from source (training) domain to a new target (test) domain such that the costly endeavour in data recollection and model rebuilding can be removed (Huang et al., 2012; Hoffman et al., 2014; Khosla et al., 2012; Torralba and Efros, 2011; Hoffman et al., 2014; Cheng et al. 2020; Kontar et al., 2020). In general, DA can be classified into semi-supervised and unsupervised DA (Ding et al., 2018). We focused on the unsupervised DA where the target data do not have labels, which is the case in our problem setting (i.e., no biopsy labels are available for the target data of a new liver).

There are generally three categories of methods in unsupervised DA (Sun et al., 2019): (1) Minimizing some distributional discrepancy measure to yield the matching between the source and target features in a common feature space. For instance, Long et al. (2015) minimized the maximum mean discrepancy (MMD) and Long et al. (2017) minimized the joint MMD between source and target domains. (2) Learning generative models to transform from the source data to the target data (Taigman et al., 2016. Hoffman et al., 2018). For instance, a direct transformation was conducted on the image pixels rather than generating a common feature space (Sun et al., 2019).

(3) Training a model on the labeled source data to estimate the pseudo-labels on the target data. Afterwards, another training was conducted on the most confident pseudo-labels of target data (Sun et al., 2019). This method was given different names such as self-ensembling (French et al., 2017) and co-training (Saito et al., 2017).

The above methods cannot achieve online DA. Online DA has gained increasing attention in recent years, where the target data is provided in a streaming manner (Hoffman et al., 2014; Bitarafan et al., 2016). Hoffman et al. (2014) posed the question of "what happens when test data not only differs from training data, but differs from it in a continually evolving way?". The authors proposed continuous domain adaptation to address the scenario of evolving domains in a classification setting. However, their method first learned domain-invariant features and then trained classifiers in two steps, which may yield sub-optimal classification accuracy.

In this paper, we propose an online DA and classification framework that can jointly learn the domain-invariant features and the classifier.

\section{Proposed Framework}

An illustration of the proposed framework is shown in Figure 2, which includes (1) graph generation and feature extraction from irregular liver surface thermal data, and (2) joint online domain adaptation and classification. Firstly, we remove the background and extract the pure liver region from the raw liver thermal images, and then 
downsample the images to reduce the dimension. Thus, the later analysis is free from the background noise but is challenged by the irregular pure liver region. To address this challenge, we construct the graph for GSP of the irregular thermal data, and then extract the features from the irregular data using GFT. Secondly, we propose a novel method to jointly perform the online DA and classification for the cross-subject liver viability classification with continuous model update. In the following, we will first introduce the GSP for irregular data analysis in Section 3.1, then describe our graph construction in Section 3.2, and finally introduce the proposed online DA and classification and its joint optimization algorithm in Sections 3.3 and 3.4, respectively.

(1) Graph Generation and Feature Extraction from Irregular Liver Surface Data

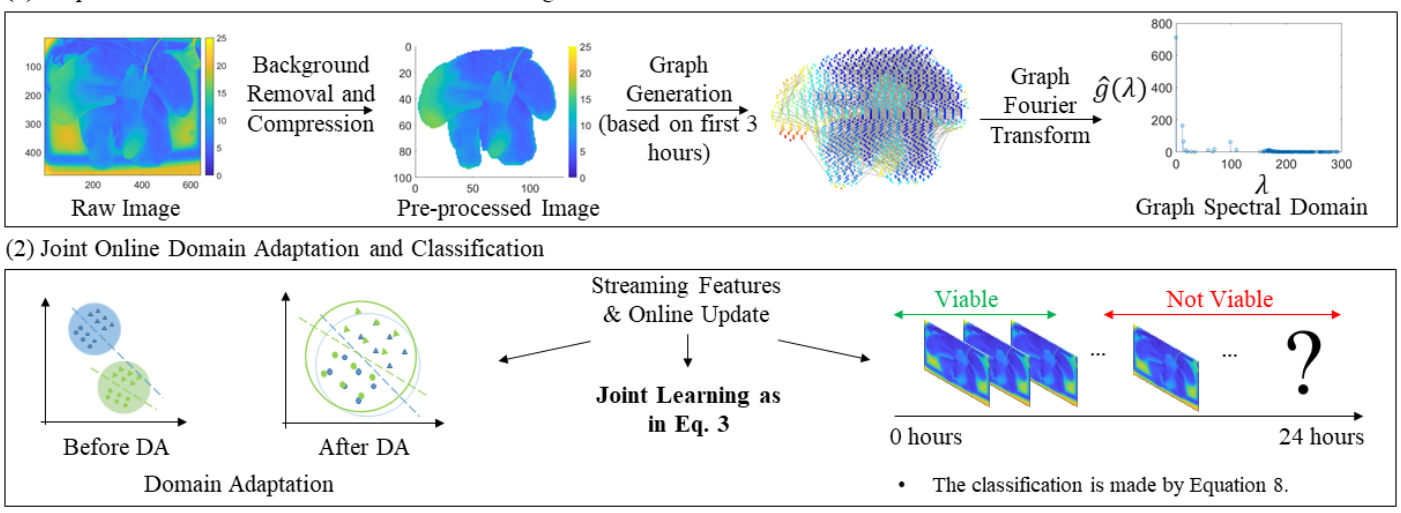

Figure 2. An illustration of the proposed framework

\subsection{Graph Signal Processing}

Throughout this paper, let $\mathcal{G}$ be a weighted graph containing a set $\mathcal{V}$ of $M$ vertices $(\mathcal{V}=$ $\left.\left\{v_{1}, \ldots, v_{M}\right\}\right)$. In our application, the vertices correspond to pixels of interest in the liver thermal data (i.e., the pixels after background removal and image compression). Let $\boldsymbol{A}$ be the symmetric adjacency matrix of the graph, where $A_{i j} \in \mathbb{R}^{+}$represents the weight between vertices $v_{i}$ and $v_{j}$. The Laplacian matrix of graph $\mathcal{G}$ is denoted by $\boldsymbol{L}$ and calculated by $\boldsymbol{L}=\boldsymbol{D}-\boldsymbol{A}$, where $\boldsymbol{D}$ denotes the diagonal matrix of degrees obtained by $\forall i: D_{i i}=\sum_{j} A_{i j} . \boldsymbol{L}$ is symmetric and real-valued and can be factorized as $\boldsymbol{L}=\boldsymbol{F} \boldsymbol{\Lambda} \boldsymbol{F}^{T}$, such that $\boldsymbol{F}$ is an orthonormal matrix, and $\boldsymbol{\Lambda}$ is a diagonal matrix, whose diagonal consists of the eigenvalues of $\boldsymbol{L}$ in ascending order.

This factorization of the Laplacian matrix serves as the building block of GFT, which extracts a spectral representation of signals situated on a graph (Ménoret et al. 2017). In particular, let $s_{t} \in \mathbb{R}^{M}$ be a vector, which denotes a realization of signal at time $t$ over the $M$ vertices of graph $\mathcal{G}$. $\boldsymbol{s}_{t}$ is a column of $\boldsymbol{S}$ (i.e., $\boldsymbol{S}_{., t}=\boldsymbol{s}_{t}$ ), which is the matrix that keeps all measurements collected throughout the experiment time, where $S_{v_{m}, t}$ denotes the temperature of vertex $v_{m}$ at time $t$.

The GFT of the signal is calculated by $\hat{\boldsymbol{s}}_{t}=\boldsymbol{F}^{T} \boldsymbol{s}_{t}$. The first elements of $\hat{\boldsymbol{s}}_{t}$, related to the lower eigenvalues in $\boldsymbol{\Lambda}$, are named low frequencies (LF) and its last elements are named high frequencies (HF). As shown in Figure 2, the HF elements are close to zero, and we consider the first $D$ LF elements to be the initial features input to DA. Actually, these LF elements preserve the main patterns in the temperature data, as shown in the case study. Let $\hat{s}_{t}^{\prime}$ be a vector with the first $D$ elements being the same as 
$\hat{\boldsymbol{s}}_{t}$, and zero elsewhere. We use $\hat{\boldsymbol{s}}_{t, 1: D}^{\prime}$ as inputs to online DA. For simplicity, we denote $\boldsymbol{f}_{t}=\hat{\boldsymbol{s}}_{t, 1: D}^{\prime}$ hereafter.

\subsection{Graph Construction}

One needs to construct the graph $\mathcal{G}$ for GFT. To preserve the main graph structure while allow the online modeling, we construct our graph as follows. Notice that GFT requires an eigendecomposition to be computed, which can be time consuming for a large matrix. We therefore construct the graph from an averaged signal $\tilde{S}_{i}=\overline{\boldsymbol{S}}_{i, 1: \tilde{t}}$ of the beginning of the data collection, so that the time-consuming eigendecompostion needs to be computed only once. Then, the weight $\boldsymbol{A}$ is defined as follows:

$$
A_{i, j}= \begin{cases}\exp \left(-\frac{\left|\tilde{S}_{i}-\tilde{S}_{j}\right|^{2}}{\sigma}\right) & i \neq j \\ 0 & i=j\end{cases}
$$

where $\sigma$ is a scale parameter. This graph can provide a good reconstruction of the irregular thermal data by using the first elements of the transformed signals $\hat{\boldsymbol{s}}_{t}$, see details in the case study.

\subsection{Online Domain Adaptation and Classification}

Let $\boldsymbol{X} \in \mathbb{R}^{N \times D}$ be the feature matrix for the graph signal $S$ of the irregular pure liver thermal data, where the $t$-th row $\boldsymbol{X}_{t, .}=\boldsymbol{f}_{t}^{T} . N$ is the number of samples (i.e., thermal images for a liver), and $D$ is the number of features (i.e., low frequency variables in GFT). Let $\boldsymbol{Y}$ be the class labels (viable or unviable) for thermal images. Adopting the notations from domain adaptation, we denote $\boldsymbol{X}_{S}$ and $\boldsymbol{X}_{T}$ as the source and target features, and $\boldsymbol{Y}_{S}$ and $\boldsymbol{Y}_{T}$ as the source and target labels. Our objective here is to: (1) train a classifier for the continuous target domain liver viability classification based on the labels in the source domain, and (2) find a subspace where the transformed features in source and target domains align well.

To achieve the objective, we propose a novel online DA and classification framework. At time $t$, we minimize the following objective function

$$
\min L\left(\phi_{t}\left(\boldsymbol{X}_{S}\right), \boldsymbol{Y}_{S}\right)+\lambda_{1} D(t)+\lambda_{2} P(t)
$$

where the first term $L\left(\phi_{t}\left(\boldsymbol{X}_{S}\right), \boldsymbol{Y}_{S}\right)$ is the classification loss at time $t, \phi(\cdot)$ is the kernel function from Reproducing Kernel Hilbert Space (RKHS) for DA. The second term $D(t)=M M D\left(\boldsymbol{X}_{S}, \boldsymbol{X}_{T_{t}}\right)=\left\|\frac{1}{N_{S}} \sum_{i=1}^{N_{S}} \phi\left(\boldsymbol{X}_{S, i}\right)-\frac{1}{N_{T_{t}}} \sum_{i=1}^{N_{T_{t}}} \phi\left(\boldsymbol{X}_{T_{t}, i}\right)\right\|$ measures the alignment of the source domain and target domain up to time $T_{t}$ in the subspace $\phi(\cdot)($ Pan et al., 2010; Long et al., 2017). Here, we will use the widely adopted Gaussian kernel for $\phi(\cdot)$. After finding the mapping to minimize MMD, the features of source and target domains are expected to be close to each other. Finally, the third term $P(t)$ is a penalty to enforce the function $\phi(\cdot)$ at two close-by time to be similar during the online DA. $P(t)=\left\|\boldsymbol{W}_{S_{t}}-\boldsymbol{W}_{S_{t-1}}\right\|_{F}^{2}=\left\|\boldsymbol{H} \boldsymbol{W}_{t}-\boldsymbol{H} \boldsymbol{W}_{t-1}\right\|_{F}^{2}$, where $\boldsymbol{W}_{t}=\left[\begin{array}{l}\boldsymbol{W}_{S_{t}} \\ \boldsymbol{W}_{T_{t}}\end{array}\right]$ is the mapping matrix in the kernel to be defined in Section 3.4, and $\boldsymbol{H}_{t}=\left[\begin{array}{ll}\boldsymbol{I}_{S \times S} & \mathbf{0}_{S \times t}\end{array}\right]$. 
In the following, we will elaborate details of each term in Equation 2 ,

Firstly, to consider the potential nonlinear relationship between the features and label, we use support vector machine (SVM) as the classifier. The SVM classification loss is,

$$
\begin{gathered}
\min L\left(\phi\left(\boldsymbol{X}_{S}\right), \boldsymbol{Y}_{S}\right)=\frac{1}{N_{S}} \sum_{i=1}^{N_{S}} \operatorname{hinge}\left(\phi\left(\boldsymbol{X}_{S, i}\right), \boldsymbol{Y}_{S, i} \mid \boldsymbol{\beta}_{t}, \beta_{0, t}\right)+\frac{\lambda}{2}\left\|\boldsymbol{\beta}_{t}\right\|^{2} \\
=\frac{1}{N_{S}} \sum_{i=1}^{N_{S}}\left(1-Y_{S, i}\left(\left\langle\boldsymbol{\beta}_{t}, \phi\left(\boldsymbol{X}_{S, i}\right)\right\rangle+\beta_{0, t}\right)\right)_{+}+\frac{\lambda}{2}\left\|\boldsymbol{\beta}_{t}\right\|^{2}
\end{gathered}
$$

where $\beta_{0, t}$ and $\boldsymbol{\beta}_{t}$ are model coefficients. According to the representer theorem (Schölkopf et al., 2001), $\boldsymbol{\beta}_{t}$ can be written as $\boldsymbol{\beta}_{t}=\alpha_{i, t} \sum_{i=1}^{N_{S}} Y_{S, i} \phi\left(\boldsymbol{X}_{S, i}\right)$. Substituting $\boldsymbol{\beta}_{t}$ into the above equation, and letting $K(a, b)=\phi(a)^{T} \phi(b)$ and $\boldsymbol{Z}_{S}=$ $\operatorname{diag}\left(Y_{S, 1}, \ldots, Y_{S, N_{S}}\right)$, we have

$$
\begin{gathered}
\min L\left(\phi\left(\boldsymbol{X}_{S}\right), \boldsymbol{Y}_{S}\right)=\frac{1}{N_{S}} \sum_{i=1}^{N_{S}}\left(1-Y_{S, i}\left(\boldsymbol{\alpha}_{t}^{T} \boldsymbol{Z}_{S} K\left(\boldsymbol{X}_{S, i}, \cdot\right)+\beta_{0, t}\right)\right)_{+}+\frac{\lambda}{2} \boldsymbol{\alpha}_{t}^{T} \boldsymbol{Z}_{S} K(\cdot, \cdot) \boldsymbol{Z}_{S} \boldsymbol{\alpha}_{t} \\
\approx \frac{1}{N_{S}} \sum_{i=1}^{N_{S}} \psi\left(Y_{S, i}\left(\boldsymbol{\alpha}_{t}^{T} \boldsymbol{Z}_{S} K\left(\boldsymbol{X}_{S, i}, \cdot\right)+\beta_{0, t}\right)\right)+\frac{\lambda}{2} \boldsymbol{\alpha}_{t}^{T} \boldsymbol{Z}_{S} K(\cdot, \cdot) \boldsymbol{Z}_{S} \boldsymbol{\alpha}_{t}
\end{gathered}
$$

where $\boldsymbol{\alpha}_{t}=\left(\alpha_{1, t}, \ldots, \alpha_{N_{s}, t}\right)^{T}$, and the $\cdot$ in $K\left(\boldsymbol{X}_{S, i}, \cdot\right)$ and $K(\cdot, \cdot)$ refers to all the possible elements. Due to the nested structure of the DA transformation and SVM parameters, the above problem is bi-convex.

We propose to develop a block-coordinate proximal gradient method based algorithm to solve the non-convex problem, which has guaranteed global convergence and high computational speed ( $\mathrm{Xu}$ and $\mathrm{Yin}, 2013)$. However, the block-coordinate proximal gradient method requires the objective to be differentiable. We therefore use the huberized SVM, a differential approximation of the SVM formulation (Wang et al. 2008), as shown in the second line of the above equation. Here, $\psi(a)= \begin{cases}0 & \text { for } a>1 \\ \frac{(1-a)^{2}}{2 \delta} & \text { for } 1-\delta<a \leq 1 \text { is derivable, and } \delta \text { is a pre-specified constant. } \\ 1-a-\frac{\delta}{2} & \text { for } a \leq 1-\delta\end{cases}$

In summary, we have the objective function at time $t$,

$$
\begin{aligned}
& \min \frac{1}{N_{S}} \sum_{i=1}^{N_{S}} \psi\left(Y_{S, i}\left(\boldsymbol{\alpha}_{t}^{T} \boldsymbol{Z}_{S} K\left(\boldsymbol{X}_{S, i}, \cdot\right)+\beta_{0, t}\right)\right)+\frac{\lambda}{2} \boldsymbol{\alpha}_{t}^{T} \boldsymbol{Z}_{S} K(\cdot, \cdot) \boldsymbol{Z}_{S} \boldsymbol{\alpha}_{t} \\
& +\lambda_{1}\left\|\frac{1}{N_{S}} \sum_{i=1}^{N_{S}} \phi\left(\boldsymbol{X}_{S, i}\right)-\frac{1}{N_{T_{t}}} \sum_{i=1}^{N_{T_{t}}} \phi\left(\boldsymbol{X}_{T_{t}, i}\right)\right\|+\lambda_{2}\left\|\boldsymbol{H} \boldsymbol{W}_{t}-\boldsymbol{H} \boldsymbol{W}_{t-1}\right\|_{F}^{2} .
\end{aligned}
$$

Next, we describe the proposed joint optimization to solve the online DA and classification in Equation 3 . 


\subsection{Joint Optimization}

Our proposed joint optimization algorithm to solve Equation 3 at time $t$ is shown in Algorithm 1. The algorithm belongs to block-coordinate proximal descent algorithm, and will iteratively solve for $\left(\boldsymbol{\alpha}_{t}, \beta_{0, t}\right)$ and $\boldsymbol{W}_{t}$ in each iteration $k$, given the source and target inputs, source labels and initial parameters. In this section, we will introduce the details of this algorithm.

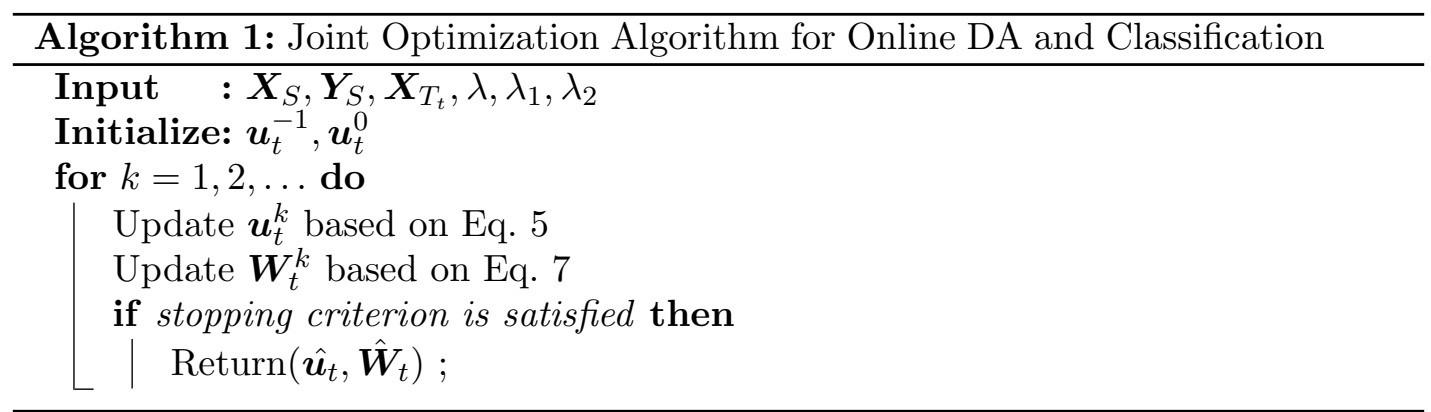

Firstly, we solve for $\left(\boldsymbol{\alpha}_{t}^{k}, \beta_{0, t}^{k}\right)$ in Equation 3 while fixing $\boldsymbol{W}_{t}^{k-1}$. In Equation 3 , only the first two terms are related to $\left(\boldsymbol{\alpha}_{t}, \beta_{0, t}\right)$, thus to solve for $\left(\boldsymbol{\alpha}_{t}^{k}, \beta_{0, t}^{k}\right)$, we have

$$
\begin{gathered}
\min _{\left(\boldsymbol{\alpha}_{t}, \beta_{0, t}\right)} \frac{1}{N_{S}} \sum_{i=1}^{N_{S}} \psi\left(Y_{S, i}\left(\boldsymbol{\alpha}_{t}^{T} \boldsymbol{Z}_{S} K\left(\boldsymbol{X}_{S, i}, \cdot\right)+\beta_{0, t}\right)\right)+\frac{\lambda}{2} \boldsymbol{\alpha}_{t}^{T} \boldsymbol{Z}_{S} K(\cdot, \cdot) \boldsymbol{Z}_{S} \boldsymbol{\alpha}_{t} \\
=\min _{\left(\boldsymbol{\alpha}_{t}, \beta_{0, t}\right)} \frac{1}{N_{S}} \mathbf{1}^{T} \boldsymbol{\psi}\left(\boldsymbol{Z}_{S} \boldsymbol{V} \boldsymbol{V}^{T} \boldsymbol{Z}_{S} \boldsymbol{\alpha}_{t}+\boldsymbol{Z}_{S} \mathbf{1} \beta_{0, t}\right)+\frac{\lambda}{2} \boldsymbol{\alpha}_{t}^{T} \boldsymbol{Z}_{S} \boldsymbol{V} \boldsymbol{V}^{T} \boldsymbol{Z}_{S} \boldsymbol{\alpha}_{t} \\
=\min _{\boldsymbol{u}_{t}} \frac{1}{N_{S}} \mathbf{1}^{T} \boldsymbol{\psi}\left(\tilde{\boldsymbol{X}}_{S} \boldsymbol{u}_{t}\right)+\frac{\lambda}{2} \boldsymbol{u}_{t}^{T} \boldsymbol{e}^{T} \boldsymbol{e} \boldsymbol{u}_{t}=\min _{\boldsymbol{u}_{t}} f\left(\boldsymbol{u}_{t}\right)+\frac{\lambda}{2} \boldsymbol{u}_{t}^{T} \boldsymbol{e}^{T} \boldsymbol{e} \boldsymbol{u}_{t}
\end{gathered}
$$

The second line is the vector form of the first line in Equation 4, where $\boldsymbol{\psi}(\boldsymbol{a})=$ $\left(\psi\left(a_{1}\right), \psi\left(a_{2}\right), \ldots, \psi\left(a_{N}\right)\right)^{T}$. In addition, the kernel matrix for the source domain $K\left(\boldsymbol{X}_{S}, \boldsymbol{X}_{S}\right)$ is approximated by $K\left(\boldsymbol{X}_{S}, \boldsymbol{X}_{S}\right)=\Phi \approx \boldsymbol{V} \boldsymbol{V}^{T}$, where $\boldsymbol{V} \in \boldsymbol{R}^{\boldsymbol{N}_{S} \times \boldsymbol{r}}$ is a rank $r$ matrix with the $i$-th row $\boldsymbol{v}_{i}$ to increase the kernel computational efficiency (Zhang et al., 2008). We further simplify Equation 4 as shown in its last line, where we denote $\boldsymbol{u}_{t}=\left(\beta_{0, t} ; \boldsymbol{\eta}_{t}\right), \boldsymbol{\eta}_{\boldsymbol{t}}=\boldsymbol{V}^{T} \boldsymbol{Z}_{S} \boldsymbol{\alpha}_{t}, \boldsymbol{e}=\operatorname{diag}\left(0, \mathbf{1}_{r \times r}\right), \tilde{\boldsymbol{X}}_{S}=\left(\boldsymbol{Z}_{S} \mathbf{1} \boldsymbol{Z}_{S} \boldsymbol{V}\right)$, and $f=\frac{1}{N_{S}} \mathbf{1}^{T} \boldsymbol{\psi}\left(\hat{\boldsymbol{X}}_{S} \boldsymbol{u}_{t}\right)$.

Equation 4 can be solved by the proximal linear update for $\boldsymbol{u}_{t}(\mathrm{Xu}$ and Yin, 2013)

$$
\begin{aligned}
\boldsymbol{u}_{t}^{k}= & \min _{\boldsymbol{u}_{t}}\left\langle\nabla f^{k}\left(\hat{\boldsymbol{u}}_{t}^{k-1}\right), \boldsymbol{u}_{t}-\hat{\boldsymbol{u}}_{t}^{k-1}\right\rangle+\frac{L_{1}^{k-1}}{2}\left\|\boldsymbol{u}_{t}-\hat{\boldsymbol{u}}_{t}^{k-1}\right\|^{2}+\frac{\lambda}{2} \boldsymbol{u}_{t}^{T} \boldsymbol{e}^{T} \boldsymbol{e} \boldsymbol{u}_{t} \\
& =\left(L_{1}^{k-1} \boldsymbol{I}_{(r+1) \times(r+1)}+\lambda \boldsymbol{e}^{T} \boldsymbol{e}\right)^{-1}\left(L_{1}^{k-1} \hat{\boldsymbol{u}}_{t}^{k-1}-\nabla f^{k}\left(\hat{\boldsymbol{u}}_{t}^{k-1}\right)\right)
\end{aligned}
$$

where $\nabla f^{k}\left(\hat{\boldsymbol{u}}_{t}^{k-1}\right)=\left(\tilde{\boldsymbol{X}}_{S}\right)^{T} \boldsymbol{\psi}^{\prime}\left(\tilde{\boldsymbol{X}}_{S} \hat{\boldsymbol{u}}_{t}^{k-1}\right), \hat{\boldsymbol{u}}_{t}^{k-1}=\boldsymbol{u}_{t}^{k-1}+w_{1}^{k-1}\left(\boldsymbol{u}_{t}^{k-1}-\boldsymbol{u}_{t}^{k-2}\right)$ is an extrapolated point, $w_{1}^{k-1}$ is the extrapolation weight, and $L_{1}^{k-1}$ is the Lipschitz constant. The extrapolation can significantly accelerate the convergence speed $(\mathrm{Xu}$ and Yin, 2013).

Secondly, we solve for $\boldsymbol{W}_{t}^{k}$, the mappings for the kernel matrix, while fixing $\left(\boldsymbol{\alpha}_{t}^{k}, \beta_{0, t}^{k}\right)$ in Equation 3. Then, Equation 3 can be converted to, 


$$
\begin{gathered}
\min _{\boldsymbol{W}_{t}} \frac{1}{N_{S}} \sum_{i=1}^{N_{S}} \psi\left(Y_{S, i}\left[\tilde{\boldsymbol{\alpha}}_{t}^{k}\right]^{T} \tilde{\boldsymbol{Z}}_{S} \boldsymbol{K} \boldsymbol{W}_{t} \boldsymbol{W}_{t}^{T} \boldsymbol{K}_{\boldsymbol{X}_{S, i}}+Y_{S, i} \beta_{0, t}^{k}\right)+\frac{\lambda}{2}\left[\tilde{\boldsymbol{\alpha}}_{t}^{k}\right]^{T} \tilde{\boldsymbol{Z}}_{S} \boldsymbol{K} \boldsymbol{W}_{t} \\
\boldsymbol{W}_{t}^{T} \boldsymbol{K} \tilde{\boldsymbol{Z}}_{S}\left[\tilde{\boldsymbol{\alpha}}_{t}^{k}\right]+\lambda_{1} \operatorname{tr}\left(\boldsymbol{K} \boldsymbol{W}_{t} \boldsymbol{W}_{t}^{T} \boldsymbol{K} \boldsymbol{S}\right)+\lambda_{2}\left\|\boldsymbol{H}_{t} \boldsymbol{W}_{t}-\boldsymbol{H}_{t} \boldsymbol{W}_{t-1}\right\|_{F}^{2}
\end{gathered}
$$

where $\tilde{\boldsymbol{\alpha}}_{t}^{k}=\left[\boldsymbol{\alpha}_{t}^{k} ; \mathbf{0}_{N_{T_{t}} \times 1}\right], \tilde{\boldsymbol{Z}}_{S}=\operatorname{diag}\left(\boldsymbol{Z}_{S}, \mathbf{0}_{N_{T_{t}} \times N_{T_{t}}}\right)$, the empirical $\tilde{\boldsymbol{K}}\left(\boldsymbol{X}_{i}, \boldsymbol{X}_{j}\right) \approx$ $\boldsymbol{K}_{\boldsymbol{X}_{i}}^{T} \boldsymbol{W}_{t} \boldsymbol{W}_{\boldsymbol{t}}^{T} \boldsymbol{K}_{\boldsymbol{X}_{j}}$, where $\boldsymbol{K}_{X}=\left[\boldsymbol{K}\left(\boldsymbol{X}_{1}, \boldsymbol{X}\right), \ldots, \boldsymbol{K}\left(\boldsymbol{X}_{S+t}, \boldsymbol{X}\right)\right]^{T}$, the empirical $\tilde{\boldsymbol{K}}=$ $\left[\begin{array}{ll}\boldsymbol{K}_{S, S} & \boldsymbol{K}_{S, t} \\ \boldsymbol{K}_{t, S} & \boldsymbol{K}_{t, t}\end{array}\right] \approx \boldsymbol{K} \boldsymbol{W}_{t} \boldsymbol{W}_{t}^{T} \boldsymbol{K}, \boldsymbol{S}=\left[\begin{array}{cc}\frac{1}{N_{S}^{2}} \boldsymbol{I}_{N_{S}} & -\frac{1}{N_{S} \times N_{T_{t}}} \boldsymbol{I}_{N_{S} \times N_{T_{t}}} \\ -\frac{1}{N_{S} \times N_{T_{t}}} \boldsymbol{I}_{N_{T_{t}} \times N_{S}} & \frac{1}{N_{T_{t}}^{2}} \boldsymbol{I}_{N_{T_{t}}}\end{array}\right]$ (Pan et al. 2010). Here, $\boldsymbol{K}_{S, S}$ is the kernel matrix of the source domain, $\boldsymbol{K}_{t, t}$ is the kernel matrix of the target domain at time $t$, and $\boldsymbol{K}_{S, t}=\boldsymbol{K}_{t, S}^{T}$ is the kernel matrix between the source and target domain at time $t$. The approximation to the empirical kernel can significantly reduce the number of parameters to be estimated, from the dimension of the sample to estimating $\boldsymbol{W}_{t}$. Then, the proximal linear update solves for

$\boldsymbol{W}_{t}^{k}=\min _{\boldsymbol{W}_{t}}\left\langle\nabla g^{k}\left(\hat{\boldsymbol{W}}_{t}^{k-1}\right), \boldsymbol{W}_{t}-\hat{\boldsymbol{W}}_{t}^{k-1}\right\rangle+\frac{L_{2}^{k-1}}{2}\left\|\boldsymbol{W}_{t}-\hat{\boldsymbol{W}}_{t}^{k-1}\right\|^{2}+\lambda_{2}\left\|\boldsymbol{H}_{t} \boldsymbol{W}_{t}-\boldsymbol{H}_{t-1} \boldsymbol{W}_{t-1}\right\|_{F}^{2}$

where $g$ is the first three items of Equation $6 . \hat{\boldsymbol{W}}_{t}^{k-1}=\boldsymbol{W}_{t}^{k-1}+w_{2}^{k-1}\left(\boldsymbol{W}_{t}^{k-1}-\boldsymbol{W}_{t}^{k-2}\right)$ is an extrapolated point, $w_{2}^{k-1}$ is the extrapolation weight and $L_{2}^{k-1}$ is Lipschitz constant.

After taking derivative of the above equation with regard to $\boldsymbol{W}_{t}$ and setting it to zero, we have the update for $\boldsymbol{W}_{t}$ as follows, see the Appendix for details.

$$
\begin{aligned}
& \boldsymbol{W}_{t}^{k}=\left(L_{2}^{k-1} \boldsymbol{I}+2 \lambda_{2} \boldsymbol{H}_{t}^{T} \boldsymbol{H}_{t}\right)^{-1}\left(L_{2}^{k-1} \hat{\boldsymbol{W}}_{t}^{k-1}+2 \lambda_{2} \boldsymbol{H}_{t}^{T} \boldsymbol{H}_{t-1} \boldsymbol{W}_{t-1}-\right. \\
& \frac{1}{N_{S}} \sum_{i=1}^{N_{S}} \psi^{\prime}\left(Y_{S, i}\left[\tilde{\boldsymbol{\alpha}}_{t}^{k}\right]^{T} \tilde{\boldsymbol{Z}}_{S} \boldsymbol{K} \hat{\boldsymbol{W}}_{t}^{k-1}\left(\hat{\boldsymbol{W}}_{t}^{k-1}\right)^{T} \boldsymbol{K}_{\boldsymbol{X}_{S, i}}+Y_{S, i} \beta_{0, t}^{k}\right) \\
& \left(\boldsymbol{K}_{\boldsymbol{X}_{S, i}} Y_{S, i}\left[\tilde{\boldsymbol{\alpha}}_{t}^{k}\right]^{T} \tilde{\boldsymbol{Z}}_{S} \boldsymbol{K}+\boldsymbol{K} \tilde{\boldsymbol{Z}}_{S}\left[\tilde{\boldsymbol{\alpha}}_{t}^{k}\right] Y_{S, i} \boldsymbol{K}_{\boldsymbol{X}_{S, i}}^{T}\right) \hat{\boldsymbol{W}}_{t}^{k-1}-\lambda\left(\boldsymbol{K} \tilde{\boldsymbol{Z}}_{S}\left[\tilde{\boldsymbol{\alpha}}_{t}^{k}\right]\right) \\
& \left.\left(\boldsymbol{K} \tilde{\boldsymbol{Z}}_{S}\left[\tilde{\boldsymbol{\alpha}}_{t}^{k}\right]\right)^{T} \hat{\boldsymbol{W}}_{t}^{k-1}-2 \lambda_{1} \boldsymbol{K} \boldsymbol{S} \boldsymbol{K} \hat{\boldsymbol{W}}_{t}^{k-1}\right)
\end{aligned}
$$

Finally, after the algorithm converges, the classification at time $t$ for the new liver viability evaluation is made by

$$
\operatorname{sign}\left(\left[\hat{\boldsymbol{\alpha}}_{t} ; \mathbf{0}_{N_{t} \times 1}\right]^{T} \tilde{\boldsymbol{Z}}_{S} \boldsymbol{K} \hat{\boldsymbol{W}}_{t} \hat{\boldsymbol{W}}_{t}^{T} \boldsymbol{K}_{\boldsymbol{X}_{S, i}}+\hat{\beta}_{0, t}\right),
$$

where $\hat{\beta}_{0, t}=\hat{\boldsymbol{u}}[1], \hat{\boldsymbol{\eta}}=\hat{\boldsymbol{u}}[2: e n d]$ and $\hat{\boldsymbol{\alpha}}_{t}=\left(\boldsymbol{K}_{S, S} \boldsymbol{Z}_{S}\right)^{-1} \boldsymbol{V} \hat{\boldsymbol{\eta}}_{t}$ 


\section{Case Study for Cross-subject Liver Viability Classification}

\subsection{Porcine Liver Procurement Data}

We applied our proposed online DA and classification framework to the cross-liver viability classification in experimental data for porcine liver procurement originally reported in Lan et al. (2015). Here, we briefly introduce the experiment and data. After being harvested from the donor, the livers were kept in the perfusion system perfused with a physiologic perfusion fluid (modified Krebs' solution). In total, there were four livers in the case study, where the perfusion temperature for Liver 1 and Liver 2 was $4^{\circ} \mathrm{C}$, while for Liver 3 and Liver 4 was $22^{\circ} \mathrm{C}$. The livers were continuously monitored with an infrared camera (FLIR Systems, Boston, MA) for 24 hours to collect the surface temperature information. In particular, the resolution of the images were $640 \times 480$ and the sampling frequency was 1 frame/minute. Every four hours, a biopsy was taken from the liver and judged by a pathologist on its viability. As reported in Lan et al. $(2015,2018)$, the livers usually became unviable after the 8-th hour while it was impossible to know the exact point where a liver changes from viable to unviable. In conformity with Lan et al. (2015, 2018), the images 3 hours before and after the 8-th hour in each liver were excluded from the classification, and a sampling frequency of 10-minute per image was used. Interested reader is referred to Lan et al. (2015, 2018) for more details.

\subsection{Data Pre-processing}

The pre-processing consists of background removal and data compression of the original liver images. For a thermal image in Figure 3 (a), firstly, we extracted the pure liver region by defining a mask, and applying the mask to the subsequent streaming images. An irregular thermal data after the background removal is shown in Figure 3 (b). Afterwards, we performed the data compression by taking a moving average of a $5 \times 5$ window. In case part of the $5 \times 5$ window falls outside of the liver region at a pixel, the average was performed based on those pixels of $5 \times 5$ window inside the liver region. As a result, Figure 3 (c) shows the compressed data for GSP, where each pixel was treated as a vertex in the graph (for instance, there were 7,756 vertices in Liver 1), and the temperature value over the vertices was a graph signal.



(a)



(b)

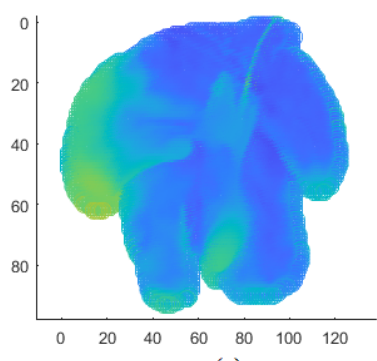

(c)

Figure 3. Data preprocessing steps: (a) Original thermal image; (b) Irregular pure liver thermal data after background removal; (c) Compressed irregular thermal data 


\subsection{Irregular Liver Thermal Data Feature Extraction using Graph Signal Processing}

We introduce the graph construction and GFT to extract the features from the irregular thermal data in this section. Firstly, to construct the graph, the images measured over the first three hours of the experiments were used to compute the averaged signal $\tilde{\boldsymbol{S}}$ in each liver. Thereafter, Equation 1 was used to obtain the $M \times M$ weight matrix. We then computed the Laplacian matrix and its factorization $\boldsymbol{L}=\boldsymbol{D}-\boldsymbol{A}=\boldsymbol{F} \boldsymbol{\Lambda} \boldsymbol{F}^{T}$. Secondly, we transformed the graph signal, using $\hat{\boldsymbol{s}}_{t}=\boldsymbol{F}^{T} \boldsymbol{s}_{t}$, to the graph spectral domain. The transformed signal $\hat{\boldsymbol{s}}_{t}$ has the same dimension as the original signal $\boldsymbol{s}_{t}$. However, as shown in Figure 2, only the first few elements of $\hat{\boldsymbol{s}}_{t}$ related to low frequencies are non-zero. In our case, the first 50 elements of $\hat{\boldsymbol{s}}_{t}$ were selected. This can significantly reduce the dimension of the problem in later analysis, while keep the patterns in the graph signal.

Figure 4 illustrates an example of the irregular thermal data reconstruction using only the first 50 elements of $\hat{\boldsymbol{s}}_{t}$. The reconstruction error can be quantified by $\frac{\left\|\left(\boldsymbol{F}^{T}\right)^{-1} \hat{\boldsymbol{s}}_{t}^{\prime}-\boldsymbol{s}_{t}\right\|_{2}}{\left\|\boldsymbol{s}_{t}\right\|_{2}}$, where $\hat{\boldsymbol{s}}_{t}^{\prime}$ is a vector with the first 50 elements being the same as $\hat{\boldsymbol{s}}_{t}$, and zero elsewhere. On average, the reconstruction error resulted by storing first 50 elements of $\hat{s}_{t}$ was 0.08 over the livers, which is reasonably small.
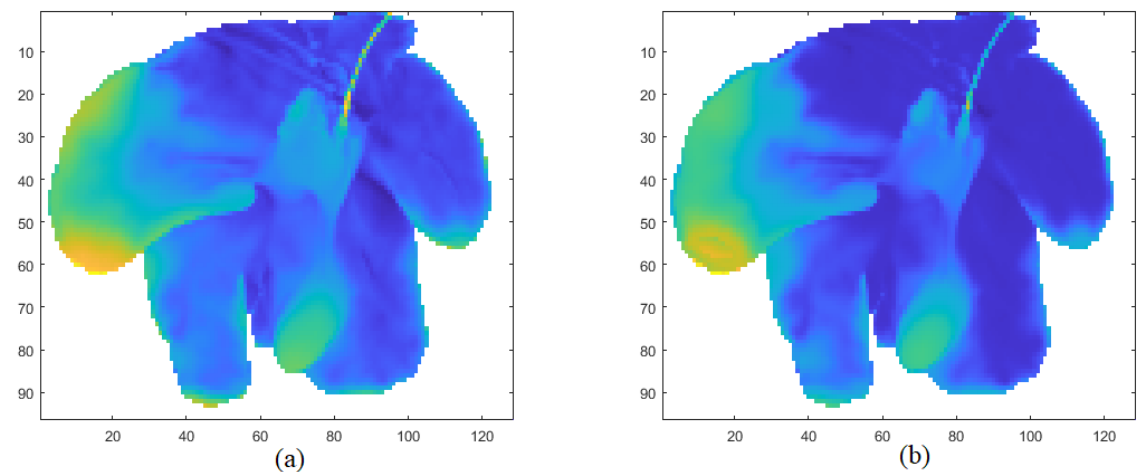

Figure 4. Illustration of irregular thermal data reconstruction of Liver 1 using: (a) All elements in $\hat{\boldsymbol{s}}_{t}$; (b) The first 50 elements in $\hat{\boldsymbol{s}}_{t}$.

\subsection{Online Domain Adaptation and Classification via Joint Optimization}

Note that in our problem, the source domain data (both irregular thermal data and viability labels) were fully available, while the irregular thermal data in the target domain (without viability labels) were observed as a continuous data stream. We continuously made the viability classification once a streaming image is observed, and updated the model every one hour. In particular, Equation 3 was hourly optimized to update the model, and predict the labels of the forthcoming images in the target domain. The model update typically took around less than 3 seconds when performed on an HP laptop equipped with a $2.10 \mathrm{GHz}$ processor and 16.0 GB of RAM running a 64-bit version of Windows 10 . Here, a hourly updated was used to balance the information accumulation and domain evolution in the target domain, and other numbers can be used in other applications.

We compare our proposed method and four benchmark methods: offline SVM, on- 
line SVM, offline subspace alignment and online subspace alignment. A leave-one-outcross-validation was used to evaluate the performance of the proposed algorithm in comparison with benchmarks, where livers were classified every 10 minutes. Specifically, in each iteration, one of the livers was considered as target and the others were considered as sources. A majority voting approach was applied, in which the final predicted label at a specific time was the one that gained more than half of the votes.

The first two benchmarks, offline SVM and online SVM, did not benefit from any DA. In offline SVM, the underlying assumption was that all of the features associated with sources and target were known. Therefore, the features could be normalized and SVM could be applied to the normalized features. However, in online SVM, we consider the streaming nature of the target data, and the features could not be normalized and SVM was directly applied to the features obtained from GFT. For the last two benchmarks, subspace alignment (SA) is an unsupervised DA method, which was originally developed for the purpose of offline DA (Fernando et al. 2013 ). In this method, the source and target features are represented by subspaces based on eigenvectors. The offline SA first perform the SA in Fernando et al. (2013), and then perform the SVM classification. The online SA considered the streaming nature of the target data, and an SA was implemented every time that a new observation was received, followed by the SVM classification (i.e., the DA and classification are done in two separate steps).

Next, we will discuss the details of the optimization process and results comparison.

\subsubsection{Details of the Optimization Process}

We set $r$ to be 50 in the approximation of $K\left(\boldsymbol{X}_{S}, \boldsymbol{X}_{S}\right)$ with $\boldsymbol{V} \boldsymbol{V}^{T}$, and our results demonstrated that the average reconstruction error associated with this factorization was $<0.03$. In Algorithm 1, Lipschitz constants and extrapolation weights were speci-

fied as $L_{1}^{k-1}=1000, L_{2}^{k-1}=1000, w_{1}^{k-1}=0.01$ and $w_{2}^{k-1}=0.01(\mathrm{Xu}$ and Yin, 2013). The $\delta$ was specified as 1 and the number of iterations was set to 50, which is sufficient for the algorithm to converge in our case study.

We set $\lambda$ to 1 and tuned the two weights associated with DA and smoothness loss functions $\left(\lambda_{1}\right.$ and $\left.\lambda_{2}\right)$ in Equation 3 via leave-one-out-cross-validation. In particular, we applied the leave-one-out-cross-validation to the source domain data at combinations of values of $\lambda_{1}$ and $\lambda_{2}$, and $\lambda_{1}$ and $\lambda_{2}$ were selected to maximize the average accuracy of the classification over all observation times and all left-out source domain data. Note that the tuning process only needs the source domain data, and can be performed offline. Here, the range of $\lambda_{1}$ and $\lambda_{2}$ are $\lambda_{1}=[20,40,60, \ldots, 200]$ and $\lambda_{2}=[0.002,0.004,0.006, \ldots, 0.020]$, respectively. The tuning parameter in SA was also selected using leave-one-out-cross-validation.

\subsubsection{Comparative Analysis}

A summary of the accuracy (ACC), precision (PRC) and recall (RCL) results for the target domain data (i.e., testing data) with different methods is given in Table1. From Table 1, when there is not any DA (offline and online SVM), the accuracy ranges from 0.75 to 0.97 for offline SVM (with an average of 0.86 ) and from 0.35 to 0.81 for online SVM (with an average of 0.67 ) across the livers. On average, the recalls associated with offline and online SVM are 0.37 and 0.25 , respectively. In both methods, there are cases in which $\mathrm{NaN}$ values are reported for precision because no livers are classified as viable in those cases. We attribute the low classification performance of these two methods to the heterogeneity of livers. However, it can be noted that normalization 
in offline SVM can slightly mitigate the liver-to-liver variation.

Table 1. Accuracy, precision and recall results for different methods

\begin{tabular}{|c|c|c|c|c|c|c|c|c|c|c|c|c|c|c|c|}
\hline \multirow{2}{*}{$\begin{array}{l}\text { Liver } \\
\text { Number }\end{array}$} & \multicolumn{3}{|c|}{$\begin{array}{c}\text { SVM } \\
\text { (offline) }\end{array}$} & \multicolumn{3}{|c|}{$\begin{array}{c}\text { SVM } \\
\text { (online) }\end{array}$} & \multicolumn{3}{|c|}{$\begin{array}{l}\text { Subspace Alignment } \\
\text { (offline) }\end{array}$} & \multicolumn{3}{|c|}{$\begin{array}{l}\text { Subspace Alignment } \\
\text { (online) }\end{array}$} & \multicolumn{3}{|c|}{$\begin{array}{c}\text { Proposed } \\
\text { (our method) }\end{array}$} \\
\hline & ACC & PRC & RCL & $\mathrm{ACC}$ & PRC & $\mathrm{RCI}$ & $\mathrm{ACC}$ & PRC & RCL & $\mathrm{ACC}$ & PRC & RCL & $1 \mathrm{CC}$ & PRC & $\mathrm{RCL}$ \\
\hline $\mathrm{L}$ & 0. & & & & & & & & & & & & & & \\
\hline & & & & & & & & & & & & & & & \\
\hline & 0.97 & 0. & & & 0 & & 0.99 & 1. & 0. & 0.76 & & & .79 & & 00 \\
\hline & & & & & & & & & & 0. & & & & & .00 \\
\hline Avg. & 0.86 & $\mathrm{NaN}$ & 0.37 & 0.67 & $\mathrm{NaN}$ & 0.25 & 0.91 & 0.89 & 0.80 & 0.85 & $\mathrm{NaN}$ & 0.34 & 0.91 & 0.75 & 1.00 \\
\hline
\end{tabular}

When the heterogeneity is addressed by DA, the accuracy ranges from 0.79 to 1.00 (with an average of 0.91) for our algorithm, from 0.76 to 0.99 (with an average of 0.85 ) for online SA and from 0.81 to 1.00 (with an average of 0.91) for offline SA across the livers. Considering accuracy, precision and recall, our algorithm outperforms its online counterpart (online SA) in accuracy and recall and shows a lower precision compared to online $\mathrm{SA}$ (in case we ignore $\mathrm{NaN}$ for online $\mathrm{SA}$ ); however, its recall is much better than that of online SA.

If we focus on the comparison between DA methods and methods without DA, it can be noted that offline SA outperforms offline SVM, and online SA and our proposed method perform better that online SVM. This result justifies the heterogeneity of the livers and highlights the necessity of using DA to alleviate this issue. A comparison between online and offline methods shows that offline methods generally outperform online ones, which is an intuitive observation. In particular, offline SVM outperforms online SVM because it benefits from scaling through normalization. Moreover, offline SA outperforms (in two out of three measures) online SA because learning the transformation is more effective when the whole target data is available and shows a comparable performance to our proposed method. Our proposed method outperforms online SA (it shows better accuracy and significantly better recall) because of the two following reasons:

- Our proposed method uses block coordinate proximal descent optimization to jointly learn the domain-invariant features and classifiers, whereas SA learns the subspaces and classifiers in a two-stage procedure.

- To regularize learning and improve performance, we use a loss function to assert that the mapping matrix changes slowly in time.

Finally, offline SA was the only method that showed a performance comparable to our proposed method. However, it is evident that an offline scenario is very idealistic and it is not applicable to real-world applications.

\section{Conclusion and Future Work}

Organ transplantation is the most effective treatment for end-stage liver disease. However, the accurate evaluation of organ viability before transplantation has been a challenging issue. Traditionally, the viability is evaluated by repetitively taking invasive biopsies on the organ. Recently, viability evaluation by infrared imaging has gained increasing interest due to its noninvasive nature. However, the existing studies used the full thermal images with background noise rather than the irregular pure liver region thermal data, and did not fully address the heterogeneity of cross-subject livers during the viability evaluation. 
In this paper, we propose a novel online DA and classification framework using the irregular thermal data for the liver viability evaluation. In particular, we extract features of irregular thermal data by GSP, and develop a joint optimization algorithm to jointly learn the domain-invariant features of the cross-subject livers and learn the classifiers. Our proposed method excels other benchmark methods, and has accurate online viability evaluations.

We will pursue several directions in the future. First, in our work, in spite of having multiple sources, we considered the problem as multiple single-source DA problems and the output prediction was done based on majority voting. In the future, we will extend our framework to the multi-source DA. In addition, there have been some works on the variance change-point detection of the original thermal images (Gao et al. 2018, 2020). We will perform the graph-based change-point detection for the irregular thermal data change-point detection.

\section{Appendix}

We provide details on the derivation of the update of $\boldsymbol{W}_{t}$ in Equation 7. Recall that the proximal update solves for

$\boldsymbol{W}_{t}^{k}=\min _{\boldsymbol{W}_{t}}\left\langle\nabla g^{k}\left(\hat{\boldsymbol{W}}_{t}^{k-1}\right), \boldsymbol{W}_{t}-\hat{\boldsymbol{W}}_{t}^{k-1}\right\rangle+\frac{L_{2}^{k-1}}{2}\left\|\boldsymbol{W}_{t}-\hat{\boldsymbol{W}}_{t}^{k-1}\right\|^{2}+\lambda_{2}\left\|\boldsymbol{H}_{t} \boldsymbol{W}_{t}-\boldsymbol{H}_{t-1} \boldsymbol{W}_{t-1}\right\|_{F}^{2}$

Taking derivative of the above equation with regard to $\boldsymbol{W}_{t}$ and setting it to zero, we have

$$
\nabla g^{k}\left(\hat{\boldsymbol{W}}_{t}^{k-1}\right)+L_{2}^{k-1}\left(\boldsymbol{W}_{t}-\hat{\boldsymbol{W}}_{t}^{k-1}\right)+2 \lambda_{2} \boldsymbol{H}_{t}^{T}\left(\boldsymbol{H}_{t} \boldsymbol{W}_{t}-\boldsymbol{H}_{t-1} \boldsymbol{W}_{t-1}\right)=0,
$$

where $\quad \nabla g^{k}\left(\hat{\boldsymbol{W}}_{t}^{k-1}\right) \quad=\quad \frac{1}{N_{S}} \sum_{i=1}^{N_{S}} \frac{\partial \psi\left(Y_{S, i}\left[\tilde{\boldsymbol{\alpha}}_{t}^{k}\right]^{T} \tilde{\boldsymbol{Z}}_{S} \boldsymbol{K} \boldsymbol{W}_{t} \boldsymbol{W}_{t}^{T} \boldsymbol{K}_{\boldsymbol{X}_{S, i}}+Y_{S, i} \beta_{0, t}^{k}\right)}{\partial \boldsymbol{W}_{t}}+$ $\frac{\lambda}{2} \frac{\partial\left(\left[\tilde{\boldsymbol{\alpha}}_{t}^{k}\right]^{T} \tilde{\boldsymbol{Z}}_{S} \boldsymbol{K} \boldsymbol{W}_{t} \boldsymbol{W}_{t}^{T} \boldsymbol{K} \tilde{\boldsymbol{Z}}_{S}\left[\tilde{\boldsymbol{\alpha}}_{t}^{k}\right]\right)}{\partial \boldsymbol{W}_{t}}+\lambda_{1} \frac{\partial\left(\operatorname{tr}\left(\boldsymbol{K} \boldsymbol{W}_{t} \boldsymbol{W}_{t}^{T} \boldsymbol{K} \boldsymbol{S}\right)\right)}{\partial \boldsymbol{W}_{t}}$. We will focus on the derivative for each of the term as follows.

According to Petersen and Pedersen (2012) Eqs. 70-72, 77,

$$
\begin{gathered}
\frac{1}{N_{S}} \sum_{i=1}^{N_{S}} \frac{\partial \psi\left(Y_{S, i}\left[\tilde{\boldsymbol{\alpha}}_{t}^{k}\right]^{T} \tilde{\boldsymbol{Z}}_{S} \boldsymbol{K} \boldsymbol{W}_{t} \boldsymbol{W}_{t}^{T} \boldsymbol{K}_{\boldsymbol{X}_{S, i}}+Y_{S, i} \beta_{0, t}^{k}\right)}{\partial \boldsymbol{W}_{t}}=\frac{1}{N_{S}} \sum_{i=1}^{N_{S}} \psi^{\prime}\left(Y_{S, i}\left[\tilde{\boldsymbol{\alpha}}_{t}^{k}\right]^{T} \tilde{\boldsymbol{Z}}_{S}\right. \\
\left.\boldsymbol{K} \boldsymbol{W}_{t} \boldsymbol{W}_{t}^{T} \boldsymbol{K}_{\boldsymbol{X}_{S, i}}+Y_{S, i} \beta_{0, t}^{k}\right)\left(\boldsymbol{K}_{\boldsymbol{X}_{S, i}} Y_{S, i}\left[\tilde{\boldsymbol{\alpha}}_{t}^{k}\right]^{T} \tilde{\boldsymbol{Z}}_{S} \boldsymbol{K}+\boldsymbol{K} \tilde{\boldsymbol{Z}}_{S}\left[\tilde{\boldsymbol{\alpha}}_{t}^{k}\right] Y_{S, i} \boldsymbol{K}_{\boldsymbol{X}_{S, i}}^{T}\right) \boldsymbol{W}_{t} \\
\frac{\lambda}{2} \frac{\partial\left(\left[\tilde{\boldsymbol{\alpha}}_{t}^{k}\right]^{T} \tilde{\boldsymbol{Z}}_{S} \boldsymbol{K} \boldsymbol{W}_{t} \boldsymbol{W}_{t}^{T} \boldsymbol{K} \tilde{\boldsymbol{Z}}_{S}\left[\tilde{\boldsymbol{\alpha}}_{t}^{k}\right]\right)}{\partial \boldsymbol{W}_{t}}=\lambda\left(\boldsymbol{K} \tilde{\boldsymbol{Z}}_{S}\left[\tilde{\boldsymbol{\alpha}}_{t}^{k}\right]\right)\left(\boldsymbol{K} \tilde{\boldsymbol{Z}}_{S}\left[\tilde{\boldsymbol{\alpha}}_{t}^{k}\right]\right)^{T} \boldsymbol{W}_{t} .
\end{gathered}
$$

According to Petersen and Pedersen (2012) Eq. 100, 


$$
\lambda_{1} \frac{\partial\left(\operatorname{tr}\left(\boldsymbol{K} \boldsymbol{W}_{t} \boldsymbol{W}_{t}^{T} \boldsymbol{K} \boldsymbol{S}\right)\right)}{\partial \boldsymbol{W}_{t}}=\lambda_{1} \frac{\partial\left(\operatorname{tr}\left(\boldsymbol{W}_{t}^{T} \boldsymbol{K} \boldsymbol{S} \boldsymbol{K} \boldsymbol{W}_{t}\right)\right)}{\partial \boldsymbol{W}_{t}}=2 \lambda_{1} \boldsymbol{K} \boldsymbol{S} \boldsymbol{K} \boldsymbol{W}_{t}
$$

Substituting the above derivative terms into Equation 9, we have

$$
\begin{gathered}
\frac{1}{N_{S}} \sum_{i=1}^{N_{S}} \psi^{\prime}\left(Y_{S, i}\left[\tilde{\boldsymbol{\alpha}}_{t}^{k}\right]^{T} \tilde{\boldsymbol{Z}}_{S} \boldsymbol{K} \hat{\boldsymbol{W}}_{t}^{k-1}\left(\hat{\boldsymbol{W}}_{t}^{k-1}\right)^{T} \boldsymbol{K}_{\boldsymbol{X}_{S, i}}+Y_{S, i} \beta_{0, t}^{k}\right)\left(\boldsymbol{K}_{\boldsymbol{X}_{S, i}} Y_{S, i}\left[\tilde{\boldsymbol{\alpha}}_{t}^{k}\right]^{T} \tilde{\boldsymbol{Z}}_{S} \boldsymbol{K}+\right. \\
\left.\boldsymbol{K} \tilde{\boldsymbol{Z}}_{S}\left[\tilde{\boldsymbol{\alpha}}_{t}^{k}\right] Y_{S, i} \boldsymbol{K}_{\boldsymbol{X}_{S, i}}^{T}\right) \hat{\boldsymbol{W}}_{t}^{k-1}+\lambda\left(\boldsymbol{K} \tilde{\boldsymbol{Z}}_{S}\left[\tilde{\boldsymbol{\alpha}}_{t}^{k}\right]\right)\left(\boldsymbol{K} \tilde{\boldsymbol{Z}}_{S}\left[\tilde{\boldsymbol{\alpha}}_{t}^{k}\right]\right)^{T} \hat{\boldsymbol{W}}_{t}^{k-1}+2 \lambda_{1} \boldsymbol{K} \boldsymbol{S} \boldsymbol{K} \hat{\boldsymbol{W}}_{t}^{k-1}+ \\
L_{2}^{k-1}\left(\boldsymbol{W}_{t}-\hat{\boldsymbol{W}}_{t}^{k-1}\right)+2 \lambda_{2} \boldsymbol{H}_{t}^{T}\left(\boldsymbol{H}_{t} \boldsymbol{W}_{t}-\boldsymbol{H}_{t-1} \boldsymbol{W}_{t-1}\right)=0
\end{gathered}
$$

Simplifying the equation, we have the update of $\boldsymbol{W}_{t}$ as shown in Equation 7

$$
\begin{gathered}
\boldsymbol{W}_{t}=\left(L_{2}^{k-1} \boldsymbol{I}+2 \lambda_{2} \boldsymbol{H}_{t}^{T} \boldsymbol{H}_{t}\right)^{-1}\left(L_{2}^{k-1} \hat{\boldsymbol{W}}_{t}^{k-1}+2 \lambda_{2} \boldsymbol{H}_{t}^{T} \boldsymbol{H}_{t-1} \boldsymbol{W}_{t-1}-\frac{1}{N_{S}} \sum_{i=1}^{N_{S}} \psi^{\prime}\left(Y_{S, i}\right.\right. \\
\left.\left[\tilde{\boldsymbol{\alpha}}_{t}^{k}\right]^{T} \tilde{\boldsymbol{Z}}_{S} \boldsymbol{K} \hat{\boldsymbol{W}}_{t}^{k-1}\left(\hat{\boldsymbol{W}}_{t}^{k-1}\right)^{T} \boldsymbol{K}_{\boldsymbol{X}_{S, i}}+Y_{S, i} \beta_{0, t}^{k}\right)\left(\boldsymbol{K}_{\boldsymbol{X}_{S, i}} Y_{S, i}\left[\tilde{\boldsymbol{\alpha}}_{t}^{k}\right]^{T} \tilde{\boldsymbol{Z}}_{S} \boldsymbol{K}+\boldsymbol{K} \tilde{\boldsymbol{Z}}_{S}\left[\tilde{\boldsymbol{\alpha}}_{t}^{k}\right] Y_{S, i}\right. \\
\left.\left.\boldsymbol{K}_{\boldsymbol{X}_{S, i}}^{T}\right) \hat{\boldsymbol{W}}_{t}^{k-1}-\lambda\left(\boldsymbol{K} \tilde{\boldsymbol{Z}}_{S}\left[\tilde{\boldsymbol{\alpha}}_{t}^{k}\right]\right)\left(\boldsymbol{K} \tilde{\boldsymbol{Z}}_{S}\left[\tilde{\boldsymbol{\alpha}}_{t}^{k}\right]\right)^{T} \hat{\boldsymbol{W}}_{t}^{k-1}-2 \lambda_{1} \boldsymbol{K} \boldsymbol{S} \boldsymbol{K} \hat{\boldsymbol{W}}_{t}^{k-1}\right) .
\end{gathered}
$$

\section{References}

Davoud Badebarin, Saeid Aslanabadi, Amir Teimouri-Dereshki, Masoud Jamshidi, Tuba Tarverdizadeh, Kaveh Shad, Kamyar Ghabili, and Ghazal Khajir. Different clinical presentations of choledochal cyst among infants and older children: A 10-year retrospective study. Medicine, 96(17), 2017.

Adeleh Bitarafan, Mahdieh Soleymani Baghshah, and Marzieh Gheisari. Incremental evolving domain adaptation. IEEE Transactions on Knowledge and Data Engineering, 28(8):2128-2141, 2016.

Ed Bullmore and Olaf Sporns. The economy of brain network organization. Nature Reviews Neuroscience, 13(5):336-349, 2012.

Andrew M Cameron and Jose F Barandiaran Cornejo. Organ preservation review: history of organ preservation. Current opinion in organ transplantation, 20(2):146$151,2015$.

CDC. Chronic liver disease and cirrhosis, 2020. URL https://www.cdc.gov/nchs/ fastats/liver-disease.htm.

Longwei Cheng, Kai Wang, and Fugee Tsung. A hybrid transfer learning framework for in-plane freeform shape accuracy control in additive manufacturing. IISE Transactions, pages 1-15, 2020.

Zhengming Ding, Sheng Li, Ming Shao, and Yun Fu. Graph adaptive knowledge transfer for unsupervised domain adaptation. In Proceedings of the European Conference on Computer Vision (ECCV), pages 37-52, 2018. 
S Feng, NP Goodrich, JL Bragg-Gresham, DM Dykstra, JD Punch, MA DebRoy, Stuart M Greenstein, and RM Merion. Characteristics associated with liver graft failure: the concept of a donor risk index. American Journal of Transplantation, 6 (4):783-790, 2006.

Basura Fernando, Amaury Habrard, Marc Sebban, and Tinne Tuytelaars. Unsupervised visual domain adaptation using subspace alignment. In Proceedings of the IEEE international conference on computer vision, pages 2960-2967, 2013.

Geoffrey French, Michal Mackiewicz, and Mark Fisher. Self-ensembling for domain adaptation. arXiv preprint arXiv:1706.05208, 2017.

Zhenguo Gao, Zuofeng Shang, Pang Du, and John L Robertson. Variance change point detection under a smoothly-changing mean trend with application to liver procurement. Journal of the American Statistical Association, 2018.

Zhenguo Gao, Pang Du, Ran Jin, John L Robertson, et al. Surface temperature monitoring in liver procurement via functional variance change-point analysis. Annals of Applied Statistics, 14(1):143-159, 2020.

Alexander M Gorbach, David B Leeser, Hengliang Wang, Douglas K Tadaki, Carlos Fernandez, David DeStephano, Douglas Hale, Allan D Kirk, Fred A Gage, and Eric A Elster. Assessment of cadaveric organ viability during pulsatile perfusion using infrared imaging. Transplantation, 87(8):1163, 2009.

Judy Hoffman, Trevor Darrell, and Kate Saenko. Continuous manifold based adaptation for evolving visual domains. In Proceedings of the IEEE Conference on Computer Vision and Pattern Recognition, pages 867-874, 2014.

Judy Hoffman, Eric Tzeng, Taesung Park, Jun-Yan Zhu, Phillip Isola, Kate Saenko, Alexei Efros, and Trevor Darrell. Cycada: Cycle-consistent adversarial domain adaptation. In International conference on machine learning, pages 1989-1998, 2018.

HRSA. Organ procurement and transplantation network: National data, 2020. URL https://optn.transplant.hrsa.gov/data/view-data-reports/ national-data/\#.

Chenhui $\mathrm{Hu}$, Xue Hua, Jun Ying, Paul M Thompson, Georges E Fakhri, and Quanzheng Li. Localizing sources of brain disease progression with network diffusion model. IEEE journal of selected topics in signal processing, 10(7):1214-1225, 2016a.

Chenhui Hu, Jorge Sepulcre, Keith A Johnson, Georges E Fakhri, Yue M Lu, and Quanzheng Li. Matched signal detection on graphs: Theory and application to brain imaging data classification. NeuroImage, 125:587-600, 2016b.

Shuai Huang, Jing Li, Kewei Chen, Teresa Wu, Jieping Ye, Xia Wu, and Li Yao. A transfer learning approach for network modeling. IIE transactions, 44(11):915-931, 2012.

Xiaoning Kang, Xiaoyu Chen, Ran Jin, Hao Wu, and Xinwei Deng. Multivariate regression of mixed responses for evaluation of visualization designs. IISE Transactions, pages 1-13, 2020.

Emmet B Keeffe. Liver transplantation: current status and novel approaches to liver replacement. Gastroenterology, 120(3):749-762, 2001.

Aditya Khosla, Tinghui Zhou, Tomasz Malisiewicz, Alexei A Efros, and Antonio Torralba. Undoing the damage of dataset bias. In European Conference on Computer Vision, pages 158-171. Springer, 2012.

Kamila Kochan, E Maslak, S Chlopicki, and M Baranska. Ft-ir imaging for quantitative determination of liver fat content in non-alcoholic fatty liver. Analyst, 140(15): 4997-5002, 2015.

Raed Kontar, Garvesh Raskutti, and Shiyu Zhou. Minimizing negative transfer of 
knowledge in multivariate gaussian processes: A scalable and regularized approach. IEEE Transactions on Pattern Analysis and Machine Intelligence, 2020.

BB Lahiri, S Bagavathiappan, T Jayakumar, and John Philip. Medical applications of infrared thermography: a review. Infrared Physics \& Technology, 55(4):221-235, 2012.

Qing Lan. Organ Viability Assessment in Transplantation based on Data-driven Modeling. PhD thesis, Virginia Tech, 2020.

Qing Lan, Ran Jin, and John L Robertson. Quantitative and qualitative evaluation for organ preservation in transplant. In IIE Annual Conference. Proceedings, page 2229. Institute of Industrial and Systems Engineers (IISE), 2015.

Qing Lan, Hongyue Sun, John Robertson, Xinwei Deng, and Ran Jin. Non-invasive assessment of liver quality in transplantation based on thermal imaging analysis. Computer methods and programs in biomedicine, 164:31-47, 2018.

Mingsheng Long, Yue Cao, Jianmin Wang, and Michael Jordan. Learning transferable features with deep adaptation networks. In International conference on machine learning, pages 97-105. PMLR, 2015.

Mingsheng Long, Han Zhu, Jianmin Wang, and Michael I Jordan. Deep transfer learning with joint adaptation networks. In International conference on machine learning, pages 2208-2217, 2017.

Mathilde Ménoret, Nicolas Farrugia, Bastien Pasdeloup, and Vincent Gripon. Evaluating graph signal processing for neuroimaging through classification and dimensionality reduction. In 2017 IEEE Global Conference on Signal and Information Processing (GlobalSIP), pages 618-622. IEEE, 2017.

Sebastian Mueller, Helmut Karl Seitz, and Vanessa Rausch. Non-invasive diagnosis of alcoholic liver disease. World journal of gastroenterology: WJG, 20(40):14626, 2014.

Antonio Ortega, Pascal Frossard, Jelena Kovačević, José MF Moura, and Pierre Vandergheynst. Graph signal processing: Overview, challenges, and applications. Proceedings of the IEEE, 106(5):808-828, 2018.

Sinno Jialin Pan, Ivor W Tsang, James T Kwok, and Qiang Yang. Domain adaptation via transfer component analysis. IEEE Transactions on Neural Networks, 22(2): 199-210, 2010.

KB Petersen and MS Pedersen. The matrix cookbook, version 20121115. Technical Univ. Denmark, Kongens Lyngby, Denmark, Tech. Rep, 3274, 2012.

Anthony Petrick, Peter Benotti, G Craig Wood, Christopher D Still, William E Strodel, John Gabrielsen, David Rolston, Xin Chu, George Argyropoulos, Anna Ibele, et al. Utility of ultrasound, transaminases, and visual inspection to assess nonalcoholic fatty liver disease in bariatric surgery patients. Obesity surgery, 25 (12):2368-2375, 2015.

Aurélie Pirayre, Camille Couprie, Frédérique Bidard, Laurent Duval, and JeanChristophe Pesquet. Brane cut: biologically-related a priori network enhancement with graph cuts for gene regulatory network inference. BMC bioinformatics, 16(1): $1-12,2015$.

Aurélie Pirayre, Camille Couprie, Laurent Duval, and Jean-Christophe Pesquet. Brane clust: Cluster-assisted gene regulatory network inference refinement. IEEE/ACM transactions on computational biology and bioinformatics, 15(3):850-860, 2017.

Jan Rothuizen and David C Twedt. Liver biopsy techniques. Veterinary Clinics: Small Animal Practice, 39(3):469-480, 2009.

Kuniaki Saito, Yoshitaka Ushiku, and Tatsuya Harada. Asymmetric tri-training for unsupervised domain adaptation. arXiv preprint arXiv:1702.08400, 2017.

Shadi Sartipi, Hashem Kalbkhani, Peyman Ghasemzadeh, and Mahrokh G Shayesteh. 
Stockwell transform of time-series of fmri data for diagnoses of attention deficit hyperactive disorder. Applied Soft Computing, 86:105905, 2020.

Bernhard Schölkopf, Ralf Herbrich, and Alex J Smola. A generalized representer theorem. In International conference on computational learning theory, pages 416426. Springer, 2001.

Justin J Skowno and Jonathan Saul Karpelowsky. Near-infrared spectroscopy for monitoring renal transplant perfusion. Pediatric Nephrology, 29(11):2241-2242, 2014.

Olaf Sporns. Networks of the Brain. MIT press, 2010.

Yu Sun, Eric Tzeng, Trevor Darrell, and Alexei A Efros. Unsupervised domain adaptation through self-supervision. arXiv preprint arXiv:1909.11825, 2019.

Yaniv Taigman, Adam Polyak, and Lior Wolf. Unsupervised cross-domain image generation. arXiv preprint arXiv:1611.02200, 2016.

Antonio Torralba and Alexei A Efros. Unbiased look at dataset bias. In CVPR 2011, pages 1521-1528. IEEE, 2011.

Hoang M Tran, Satish TS Bukkapatnam, and Mridul Garg. Detecting changes in transient complex systems via dynamic network inference. IISE Transactions, 51 (3):337-353, 2019.

E Vazquez-Martul and JC Papadimitriou. Importance of biopsy evaluation and the role of the pathologist in solid organ transplant programs. In Transplantation proceedings, volume 3, pages 725-728, 2004.

Enrico Vidal, Angela Amigoni, Valentina Brugnolaro, Giulia Ghirardo, Piergiorgio Gamba, Andrea Pettenazzo, Giovanni Franco Zanon, Chiara Cosma, Mario Plebani, and Luisa Murer. Near-infrared spectroscopy as continuous real-time monitoring for kidney graft perfusion. Pediatric Nephrology, 29(5):909-914, 2014.

Michael L Volk, Heidi A Reichert, Anna SF Lok, and Rodney A Hayward. Variation in organ quality between liver transplant centers. American Journal of Transplantation, 11(5):958-964, 2011.

Li Wang, Ji Zhu, and Hui Zou. Hybrid huberized support vector machines for microarray classification and gene selection. Bioinformatics, 24(3):412-419, 2008.

Yangyang $\mathrm{Xu}$ and Wotao Yin. A block coordinate descent method for regularized multiconvex optimization with applications to nonnegative tensor factorization and completion. SIAM Journal on imaging sciences, 6(3):1758-1789, 2013.

Kai Zhang, Ivor W Tsang, and James T Kwok. Improved nyström low-rank approximation and error analysis. In Proceedings of the 25th international conference on Machine learning, pages 1232-1239, 2008. 